\title{
Ethylene Glycol as an Entrainer in the Extractive Distillation of Acetonitrile + Water System: Simulation
}

\author{
Dhoni Hartanto ${ }^{1}$, Waliyuddin Sammadikun ${ }^{2}$, Widi Astuti ${ }^{3}$, Irene Nindita Pradnya ${ }^{4}$, Maharani \\ Kusumaningrum $^{5}$, Ria Wulansarie ${ }^{6}$, Ianatul $\mathrm{Khoiroh}^{7}$, Achmad Chafid $^{8}$ \\ \{dhoni.hartanto@mail.unnes.ac.id ${ }^{1}$,waliyudin7@gmail.com², widi_astuti@mail.unnes.ac.id ${ }^{3}$ \} \\ Department of Chemical Engineering, Faculty of Engineering, Universitas Negeri Semarang, Kampus \\ Sekaran Gunungpati, Semarang, 50229, Indonesia ${ }^{1}$, Department of Chemical and Environmental \\ Engineering, Faculty of Engineering, University of Nottingham Malaysia Campus, Jalan Broga, \\ Semenyih, 43500 Selangor Darul Ehsan, Malaysa ${ }^{2}$, Department of Chemical Engineering, Universitas \\ Islam Indonesia, Yogyakarta, 55584, Indonesia $^{3}$
}

\begin{abstract}
Acetonitrile is one of the important organic solvent which widely used in the industrial application. The high purity of acetonitrile can be obtained with the extractive distillation process to separate acetonitrile from water by using an entrainer. The acetonitrile-water extractive distillation using ethylene glycol as an entrainer was investigated using Aspen Plus. The RadFrac module distillation followed by ethylene glycol recovery column was utilised in the simulation. The phase equilibrium calculation of the mixture was calculated using The Non-Random Two Liquids-Hayden-O'Connell (NRTL-HOC) thermodynamic model. The effects of reflux ratio, number of stages, binary and entrainer feed stage, feed temperature on the distillate composition and energy consumption were discussed. The results show that the optimum parameters design consists of the theoretical stages of 45 , the binary feed stage of 27 , entrainer feed stage of 15 , the binary feed temperature of $62{ }^{\circ} \mathrm{C}$, and the reflux ratio of 0.6 were required to achieve the purity of acetonitrile higher than $99 \% \mathrm{w}$.
\end{abstract}

Keywords: acetonitrile, extractive distillation, entrainer, ethylene glycol, simulation

\section{Introduction}

Acetonitrile is one of the organic compounds and used intensively as solvents in the pharmaceutical industry. Acetonitrile was also used in Gas Chromatography (GC), Ultraviolet (UV) analysis, Thin Layer Chromatography (TLC), High-Performance Liquid Chromatography (HPLC), and drugs analysis. The wide application causes the demand for acetonitrile reaches $70 \%$ of all ingredients in the pharmaceutical industry [1]. Acetonitrile has several advantages such as the ability to dissolve polar and non-polar compounds, low boiling points and freezing points, low viscosity, and non-toxic material [2].

Acetonitrile can be obtained from the byproduct of acrylonitrile production using ammoxidation process of propylene with ammonia and oxygen [3]. However, byproduct contains various contaminants such as hydrogen cyanide, water, acrylonitrile, acetaldehyde, oxazole, cisand trans-crotononitrile, metacrylonitrile, acetone, methanol, acrolein, and alcoholic allyl. The initial composition of crude acetonitrile was 52\% acetonitrile, $43.6 \%$ water, $2.5 \%$ hydrogen 
cyanide, $0.5 \%$ acrylonitrile, and $1.3 \%$ other impurities [3]. The mixture is then purified to meet a high purity grade.

The first purification process is carried out by a batch process. In this process, the crude acetonitrile is distilled and reacted with a strong base mixture, sodium hydroxide, and formaldehyde. The product from this process is an acetonitrile-water mixture which exhibited the azeotropic point [4]. The mixture of acetonitrile-water has a minimum boiling point azeotropic of $349.92 \mathrm{~K}$ at $x_{1}=0.683$ [5]. Separation of the mixture is difficult by conventional distillation methods due to azeotrope point.

Several modifications to the distillation system have been carried out to overcome the present of azeotrope in the system such as pressure-swing distillation [6], azeotropic distillation [7], and extractive distillation [8]. Pressure-swing distillation uses two different pressure columns to separate the azeotropic point. Azeotropic distillation and extractive distillation both use a third component called entrainer. Azeotropic distillation uses a light entrainer so the component and entrainer will be the top product, but azeotropic distillation requires large additional energy to vaporize the mixture [9]. Extractive distillation uses a heavy entrainer that is able to alter the relative volatility of the component. Pure components are produced as top products, and no solvent evaporation process is required as in azeotropic distillation. For this reason, extractive distillation is more recommended than azeotropic distillation [10].

Extractive distillation can be an alternative distillation technique to a system that has azeotropic points like acetonitrile/ water. In extractive distillation, the entrainer is added at the top stage of the distillation column. The selected entrainer must be compatible with the azeotropic binary system to be able to break the azeotropic point. In addition, entrainers must have stable properties, high selectivity, and low toxicity [11]. Some examples of entrainers for extractive distillation such as water [12], dimethyl sulfoxide [13], glycerol [14], mixtures of organic solvents and ionic liquids [15], biological buffer tris (hydroxymethyl) aminomethane (TRIS) [16], 1,2propanadiol [17].

Several studies were conducted for acetonitrile/water azeotropic systems using various entrainers. Zhang et al. (2013) used dimethyl sulfoxide (DMSO) as an entrainer [13]. The acetonitrile/water azeotropic point was broken at 0.4 of mole fraction of DMSO. DMSO has a high polarity, so it forms hydrogen bonds with water that stronger than those existed in the acetonitrile/water system. In other studies, butyl acetate can be used as an entrainer [18]. However, butyl acetate is less effective because it has low boiling points that difficult to be recovered [13]. In addition, butyl acetate will form new azeotropes with water [19].

Another potential entrainer for a non-ideal system in extractive distillation is ethylene glycol. Ethylene glycol has characteristics such as low vapor pressure, low viscosity, and low toxicity [5]. Several studies were conducted to know the performance of ethylene glycol as an entrainer. Dong et al (2018) [20] reported that 0.2 of mole fraction of ethylene glycol was able to eliminate the azeotropic point of the methylal/methanol system. The ethanol dehydration process can also be optimized using ethylene glycol. Kamihama et al. (2012) reported that 0.059 of mole fraction of ethylene glycol can produce high ethanol purity [21]. You et al. (2018) [22] also study the performance of ethylene glycol in acetonitrile/water system. The operating pressure was conducted lower than $101.325 \mathrm{kPa}$ using three column consist of preconcentration, extractive, and regeneration column with an initial acetonitrile mol fraction of 0.2 . The purity of acetonitrile in the preconcentration column is $65 \%$.

In this study, the effect of stages, reflux ratio (RR), entrainer stage, binary feed stage (BFS) to product composition, reboiler and condensor duty, and energy duty were investigated using just two columns at $101.325 \mathrm{kPa}$, extractive, and regeneration column with initial concentration 
acetonitrile as $0.45 \mathrm{~mol}$ fraction. The NRTL-HOC model was used as a thermodynamic package in the simulation.

\section{Simulation of the acetonitrile dehydration extractive distillation}

The simulation was conducted using two column consists of extractive distillation and entrainer recovery column. The main flowsheet of this simulation was presented in Fig. 1.

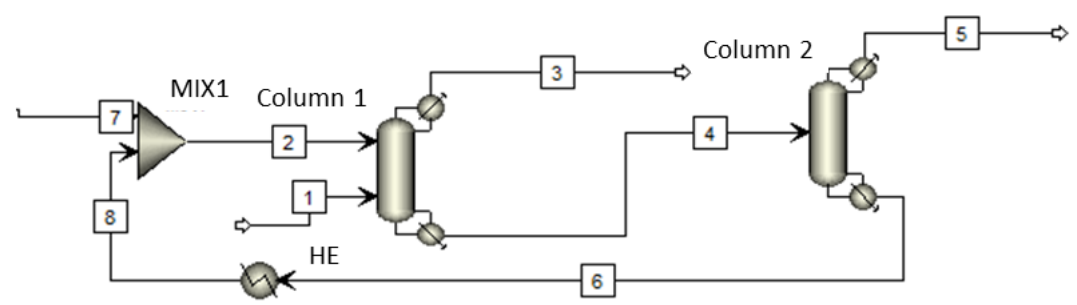

Figure.1. The extractive distillation process flow diagram of acetronitrile purification using ethylene glycol as an entrainer.

The isobaric vapor-liquid equilibria (VLE) data of acetonitrile-water-ethylene glycol system was obtained from the literature [5]. The extractive distillation was simulated using The NRTL-HOC thermodynamic model. The optimum binary interaction parameters of acetonitrile-water, acetonitrile-ethylene glycol, and water-ethylene glycol were obtained from Aspen Plus physical property databank, which shown in Table 1.

Table 1. Binary Interaction Parameters.

\begin{tabular}{lccc}
\hline \multicolumn{1}{c}{ Component } & \multicolumn{3}{c}{ Parameters } \\
\cline { 2 - 4 } & $M i j$ & Water & $M i j$ \\
\hline Acetonitirle-water & -0.1164 & 1.0567 & 0.3 \\
Acetonitrile-ethyelen glicol & 0 & 0 & 0.3 \\
Water-acetonitrile & 0.3479 & -0.0567 & 0.3 \\
\hline
\end{tabular}

The total pressure and vapor pressure of the component were calculated using the extended Antoine, as can be seen in equation (1). The extended Antoine constant values listed in Table 2 with the unit pressure and temperature are $k P a$ and $K$, respectively.

$$
\ln P^{s}=A_{1}+A_{2} /\left(T+A_{3}\right)+A_{4} T+A_{5} \ln T+A_{6} T^{A_{7}} \text { for } A_{8}<T<A_{9}
$$


Table 2. Extended Antoine Parameters*.

\begin{tabular}{lccc}
\hline \multirow{2}{*}{ Parameters } & \multicolumn{3}{c}{ Compound } \\
\cline { 2 - 4 } & Acetonitrile & Water & ethylene glycol \\
\hline$A_{1}$ & 35.2221 & 62.1361 & 72.5771 \\
$A_{2}$ & -5126.2 & -7258.2 & -10411 \\
$A_{3}$ & 0 & 0 & 0 \\
$A_{4}$ & 0 & 0 & 0 \\
$A_{5}$ & -3.5406 & -7.3037 & -8.1976 \\
$10^{-15} A_{6}$ & $1.3995 \times 10^{-17}$ & $4.1653 \times 10^{-6}$ & $1.6536 \times 10^{-18}$ \\
$A_{7}$ & 6 & 2 & 6 \\
$A_{8}$ & 229.32 & 273.16 & 260.15 \\
$A_{9}$ & 545.5 & 647.1 & 720 \\
\hline \multicolumn{3}{c}{$*$ Taken from Aspen Plus physical property databank }
\end{tabular}

\section{Result and Discussion}

\subsection{Process Design Parameters}

In this work number of stages, reflux ratio, binary feed stage, and entrainer feed stage were analyzed. The optimum binary and entrainer feed stage to obtain the purity of acetronitrile of $99 \% \mathrm{w}$ were 27 and 4, respectively. Theoretical stages of 45 were used as an optimum stage number in the extractive distillation column simulation. The optimum amount of entrainer which added into the system to break the azeotrope of acetonitrile-water is 0.277 mol fraction [5]. Process design parameters used in the simulation are Feed flowrate of 150 $\mathrm{kmol} / \mathrm{h}$; Distillate mole flow $60 \mathrm{kmol} / \mathrm{h}$; Acetonitrile feed mole fraction of 0.45 ; Theoritical stage numbers of 45; Entrainer mole fraction of 0.227 ; Feed temperature of $25^{\circ} \mathrm{C}$; Entrainer temperature of 25 ${ }^{\circ} \mathrm{C}$; Binary feed stage of 27; Entrainer feed stage of 4; and Pressure $101.325 \mathrm{kPa}$.

\subsection{Sensitivity}

Figure 2 shows the effect of the reflux ratio on the purity of acetonitrile. The commercial specification of acetonitrile of $99 \% \mathrm{w}$ [23] can be achieved using refluxes among 0.6 and 1.5. As the reflux ratio increase, the purity of acetonitrile increase. In the McCabe-Thiele method, the increasing of reflux ratio (RR) will be followed by the increasing of the distillate concentration $\left(x_{\mathrm{D}}\right)$ [24]. Nevertheless, an increase of reflux ratio greater than 0.8 causes a decrease in the distillate. At these refluxes, the dilution of the entrainer occurred, thus the effect on the relative volatility became less [10]. 


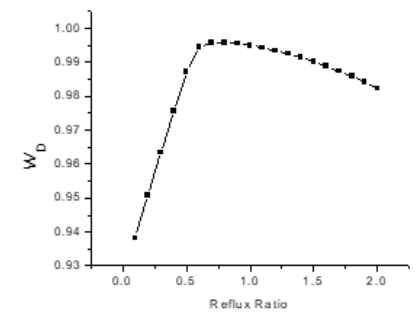

Figure.2. Reflux ratio (RR) effect on the mass fraction of acetonitrile $\left(w_{D}\right)$ (Number of stages: 45 , Entrainer Feed Stage: 4, Binary Feed Stage: 27)
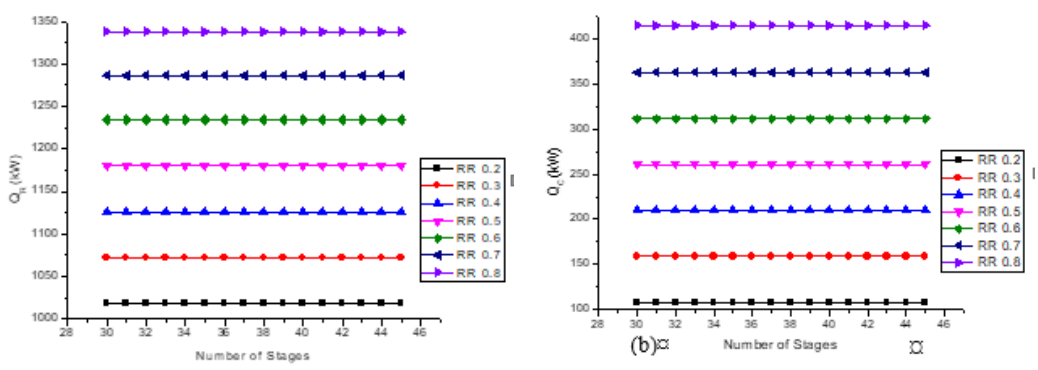

Figure. 3. (a) Number of stages and reflux ratio effect on the reboiler duty $\left(Q_{R}\right)$; (b) Number of stages and reflux ratio effect on the condenser duty $\left(Q_{C}\right)$. (Entrainer Feed Stage: 4, Binary Feed Stage: 27$)$.

Figure 3 show the influence of the number of stages and reflux ratio on the reboiler and condenser duties. According to both figures, the number of stages did not have a significant impact on energy consumption. However, the reflux ratio had a significant effect on the reboiler $\left(Q_{R}\right)$ and condenser $\left(Q_{C}\right)$ duties. Heating and cooling requirements presented a directly proportional relationship with the reflux ratio [12]. In this simulation, the pure acetonitrile and the energy requirements are satisfied with 30 stages and are operated at reflux ratio of 0.6 .
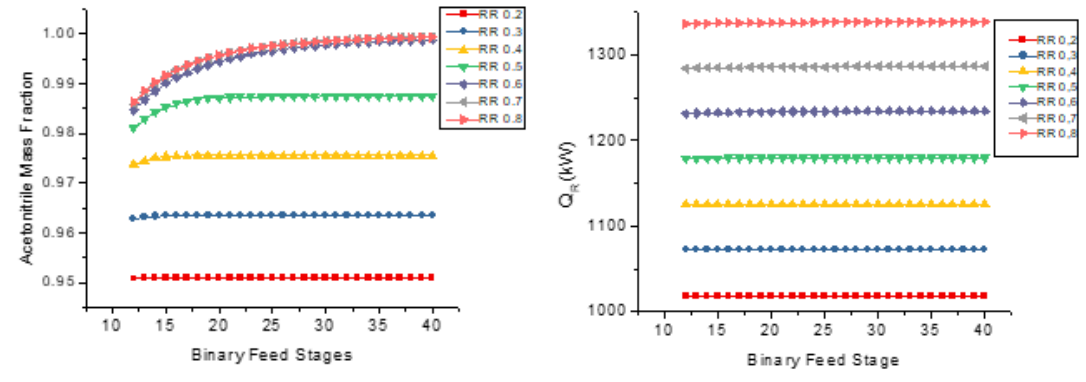

Figure. 4. (a) Binary feed stage effect on the distillate composition; (b) Binary feed stage on the reboiler duty 
Figure 4 (a) presents the simulation's results between analysis of the reflux ratio and the binary feed stage to acetonitrile mass fraction. The best result of distillate composition was obtained at reflux ratio between $0.6-0.8$. At reflux ratio under 0.6 , the result of acetonitrile mass fraction showed an impairment which has the highest purity only 0.987 . The best result of distillate acetonitrile showed when the binary mixture was fed at stage 15 with a value higher than 0.99 . Figure 4 (b) shows the influence of various ratio reflux and binary feed stages to reboiler energy consumption. The increasing ratio reflux required higher energy consumption, with different value for each reflux ratio reached between $52-53 \mathrm{~kW}$. But at the same reflux ratio, energy consumption relatively constant to the binary feed stages. The optimum reboiler energy consumption was obtained in the 0.6 ratio reflux which produced $0.99 \%$ w of acetonitrile.

Energy consumption was also analyzed to determine the best configuration of binary and entrainer feed stage at reflux ratio 0.6. Figure 4 shows the influence of feed stage configuration on the distillate composition and energy.
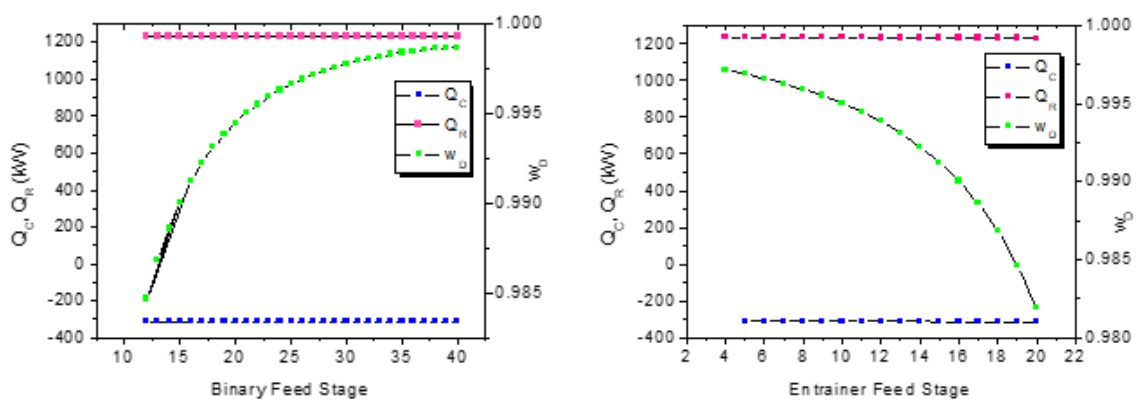

Figure. 5. (a) Effect of Binary Feed Stage on Distillate Composition and Reboiler and Condenser Duty (RR 0.6, EFS 4); (b) Effect of Entrainer Feed Stage on Distillate Composition and Reboiler and Condenser Duty (RR 0.6, BFS 26).

Figure 5 (a) shows the binary feed stage on the acetonitrile mass fraction and energy duties of condensor and reboiler at the constant ratio reflux (0.6). At any binary feed stage, both condenser and reboiler energy consumption remains constant. The distillate concentration give different behaviour. It increased inline with the binary feed stage increasing. At stage of 16 , the purity started to have a higher value than 0.99 . Hence, it could determine the optimum energy duty at stage 16. The optimum energy duties for condensor and reboiler were -312.480 and $1232.710 \mathrm{~kW}$, respectively. Figure 5 (b) presents the entrainer feed stage on the acetonitrile mass fraction and energy duties of condensor and reboiler at the constant ratio reflux (0.6). The characteristic of energy duties for both condensor and reboiler showed constantly. The concentration of acetonitrile is decreasing as increasing of entrainer feed stage. The purity was begun to have more than 0.99 when it was at stage 4 . But the energy duties were not satisfied. The optimum energy duties with higher purity than 0.99 met at stage 15 . The energy at stage of 15 for condensor and reboiler were $-312.324 \mathrm{~kW}$ and $1232.539 \mathrm{~kW}$, respectively.

Comparing to both figures, the best configuration based on total energy consumption is a binary feed stage of 27 and entrainer feed stage of 15 . 


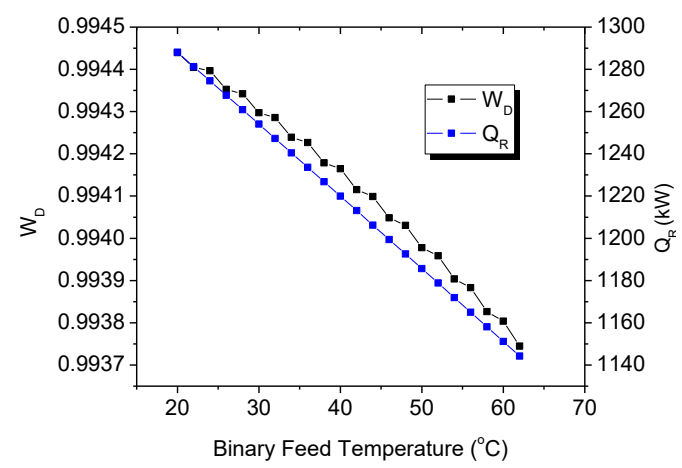

Figure. 6. The effect of binary feed temperature on the distillate composition and reboiler duty.

Figure 6 shows the influence of feed temperature on the distilate composition and reboiler. A rise of feed temperature from $20-62{ }^{\circ} \mathrm{C}$ decreased the acetonitrile composition in the distillate as well as the decreasing of the reboiler duty. The higher feed temperature, the less energy required to vaporize the liquid. The optimum binary feed temperature was $62{ }^{\circ} \mathrm{C}$. The effect of feed temperature on the condenser duty was not significant.

\section{Conclusion}

The extractive distillation of acetonitrile-water system using ethylene glycol as an entrainer was simulated using Aspen Plus. The increasing of reflux ratio until 0.6 resulting in the increasing of acetonitrile purity as well as energy consumption. On the other hand, the number of stages did not affect energy consumption. Binary feed stage gave a constant effect to the energy consumption of boiler and condensor but the increasing of binary feed stage yielding an increasing of distillate concentration. The increasing of entrainer feed stage decreased the concentration of acetonitrile. Moreover, the increasing of feed temperature decreased the acetonitrile compositions and reboiler duty. In order to produce acetonitrile with the purity higher than $99 \% \mathrm{w}$, the optimum parameters design were theoretical stages of 45 , followed by the binary and entrainer feed stages at 27 and 15, respectively. The optimum temperature of the binary feed was $62{ }^{\circ} \mathrm{C}$, and the optimum reflux ratio was 0.6 . 


\section{References}

[1] Bonilla, R.: Cost effective solutions to the world wide MeCN shortage. http://www.pharmpro.com/articles/2010/04/Cost-Effective-Solutions-to-the-World-WideMeCNShortage/ (accessed 10/11/18). (2010)

[2] Mcconvey, F.I., Woods, D., Lewis, M., Gan, Q., and Nancarrow, P.: The importance of acetonitrile in the pharmaceutical industry and opportunities for its recovery from waste. Organic Process Research \& Development. pp. 2-41 (2012)

[3] Cesa, M. C., Jacobson, P. A., and Wappelhorst, R. L.: Purification of acetonitrile by a distillative recovery/ion exchange resin treatment process. Standard Oil Co. EP0890572A1 (1997)

[4] Fitzgibbons, William O., Barko, and Andrew J.: Removal of cyanides from acetonitrile. Standard Oil Company. US4328075 (1982)

[5] Zhang, L., Shen, D., Zhang, and Wu, X.: Experimental measurement and modelling of vaporliquid equilibirum for the ternatry system water+acetonitrile+ethylene glycol. Journal of Chemical dan Engineering, 61(7). pp. 2596-2604 (2017)

[6] Luyben, W. L. Pressure-swing distillation for minimum- and maximum-boiling homogeneous azeotropes. Industrial dan Engineering Chemistry Research, 51(33). pp. 10881-10886 (2012)

[7] Widagdo, S., and Seider, W.D., Azeotropic Distillation. AICHE Journal. 42(1). pp. 96-130 (1996)

[8] Lei, Z., Li, C., and Chen, B.: Extractive distillation: a review. Separation dan Purification Reviews, 32(2). pp. 121-213 (2003)

[9] Ma, S. Hou, Y., Sun, Y., Li, J., Li, Y., Sun, L.: Simulation and experiment for ethanol dehydration using low transition temperature mixtures (LTTMs) as entrainers. Chemical Engineering and Processing: Process Intensification, 121.pp. 71-80 (2017)

[10] Meirelles, A.: Ethanol dehydration by extractive distillation. Chemical Techonogy Biotechnology, 53. pp. 181-188 (2007)

[11] Lladosa, E., Monton, J.B., Burguet, M.C., and Munoz, R.: Phase equilibria involved in extractive distillation of dipropyl ether+1-propyl alcohol using 2-ethoxyethanol as entrainer. Fluid Phase Equilibria, 255(1). pp. 62-69 (2007)

[12] Gil, I.D., Botia, D.C., Ortiz, P., and Sanchex, O.F.: Extractive distillation of acetone/methanol mixture using water as entrainer. Industrial Engineering Chemistry Research, 48.pp. 4858-4865 (2009)

[13] Zhang, Z., Huang, D., Ming, Jia, P., Sun, D., Li, W.: Isobaric vapor-liquid equilibrium for the extractive distillation of acetonitrile + water mixtures using dimethyl sulfoxide at $101.3 \mathrm{kPa}$. Journal of Chemical dan Engineering Data, 58(12). pp. 3364-3369 (2013)

[14] Zhang, Z., Huang, D., Ming, Jia, P., Sun, D., Li, W.: Entrainer selection for separating tetrahydrofuran/water azeotropic mixture by extractive distillation. Separation and Purification Technology, 122. pp. 73-77 (2014)

[15] Dai, C., Lei, ., Xi, X., Zhu, J., and Chen, B.: 2014. Extractive distillation with a mixture of organic solvent and ionic liquid as entrainer. Industrial dan Engineering Chemistry Research, 53(40). pp. 15786-15791 (2014)

[16] Hartanto, D., Gupta, B. S., Taha, M., and Lee, M.-J. Isobaric vapour-liquid equilibrium of (tert-butanol+water) system with biological buffer TRIS at $101.3 \mathrm{kPa}$. The Journal of Chemical Thermodynamics, 98. pp. 159-164 (2016)

[17] $\mathrm{Xu}, \mathrm{S}$., and Wang, H.: A new entrainer for separation of tetrahydrofuran-water azeotropic mixture by extractive distillation. Chemical Engineering and Processing: Process Intensification, 45(11). pp. 954-958 (2006)

[18] Acosta, J., Arce, A., Rodil, E., and Soto, A.. A thermodynamic study on binary and ternary mixtures of acetonitrile, water and butyl acetate. Fluid Phase Equilibria, 203(1). pp. 83-98 (2002)

[19] Sazonova, A., and Raeva, V.M. 2015. Recovery of acetonitrile from aqueous solutions by extractive distillation effect of entrainer. International Journal of Chemical Nuclear, Metallurgical and Materialss Engineering, 9(2). (2015)

[20] Dong, Y., Dai, C., and Lei, Z.: Extractive distillation of methylal/methanol mixture using ethylene glycol as entrainer. Fluid Phase Equilibria, 462. pp. 172-180 (2018) 
[21] Kamihama, N., Matsuda, H., Kurihara, K., Tochigi, K. and Oba, S.: Isobaric Vapor-Liquid Equilibria for Ethanol + Water + Ethylene Glycol and Its Constituent Three Binary Systems. Journal of Chemical dan Engineering Data, 57(2). pp. 339-344 (2012)

[22] You, X., Gu, J., Gerbaud, V., Peng, C., Liu, H.:Optimization of pre-concentration, entrainer recycle and pressure selection for the extraction distillation of acetonitrile-water with ethylene glycol. Chemical Egnineering Science. (2017)

[23] Grayson M.: Kirk-Othmer concise encyclopedia of chemical technology.Tokyo, Maruzen Co. pp. 997-998 (1985)

[24] Petlyuk, F. B.: Distillation theory and its application to optimal design of separation units. Cambridge University Press. UK (2004) 